\title{
The use of a single daily dose of tadalafil to treat signs and symptoms of benign prostatic hyperplasia and erectile dysfunction
}

\author{
Mauro Gacci' \\ Matteo Salvi ${ }^{\prime}$ \\ Arcangelo Sebastianelli ${ }^{\prime}$ \\ Linda Vignozzi ${ }^{2}$ \\ Giovanni Corona ${ }^{3}$ \\ Kevin T McVary ${ }^{4}$ \\ Steven A Kaplan ${ }^{5}$ \\ Mario Maggi \\ Marco Carini ${ }^{\prime}$ \\ Matthias Oelke 6 \\ 'Department of Urology, University \\ of Florence, Florence, ${ }^{2}$ Sexual \\ Medicine and Andrology Unit, \\ Department of Experimental \\ and Clinical Biomedical Sciences, \\ University of Florence, Florence, \\ ${ }^{3}$ Endocrinology Unit, Maggiore-Bellaria \\ Hospital, Bologna, Italy; ${ }^{4}$ Division \\ of Urology, Department of Surgery, \\ Southern Illinois University School of \\ Medicine, Springfield, IL, ${ }^{5}$ Department \\ of Urology, Weill Cornell Medical \\ College, Cornell University, New York, \\ NY, USA; ${ }^{6}$ Department of Urology, \\ Hannover Medical School, Hannover, \\ Germany
}

This article was published in the following Dove Press journal:

Research and Reports in Urology

25 April 2013

Number of times this article has been viewed

\begin{abstract}
A strong and independent association between lower urinary tract symptoms suggestive of benign prostatic hyperplasia (LUTS/BPH) and erectile dysfunction (ED) has been widely evidenced in several clinical epidemiologic studies. Preclinical animal models have provided a great deal of information on potential common pathogenic mechanisms underlying these two clinical identities. Although the efficacy of the most commonly used treatments for LUTS/BPH is well defined, the negative impact of these treatments on sexual function - in particular, on ED - has triggered the search for new treatment options. In this regard, a new role for phosphodiesterase type 5 inhibitors in the treatment of LUTS/BPH and ED has been claimed. Tadalafil is one of the most extensively investigated phosphodiesterase type 5 inhibitors for this new indication. All evidence reported to date suggests that tadalafil $5 \mathrm{mg}$ once daily is a safe and effective treatment option for both LUTS/BPH and ED.
\end{abstract}

Keywords: phosphodiesterase type 5 inhibitor, LUTS/BPH, ED, sexual function, Cialis

\section{Introduction}

During the last decade, several preclinical and clinical studies have been initiated to investigate the links between lower urinary tract symptoms suggestive of benign prostatic hyperplasia (LUTS/BPH) and erectile dysfunction (ED). ${ }^{1}$ The reported evidence is based on single and multicenter trials from general and/or uro-/andrological populations; however, trial outcomes have been controversial.

Although the efficacy of all currently available treatments for LUTS/BPH is well defined, the negative impact of all therapies on erectile function (EF) is still under evaluation. Lifestyle modifications and phytotherapies have no or minimal impact on sexual function but these are also less effective on lower urinary tract symptoms (LUTS). In contrast, $\alpha$-blockers, $5 \alpha$-reductase inhibitors, and prostatic surgery, although associated with a strong improvement in LUTS, are usually associated with worsening sexual function. ${ }^{1}$

Several clinical trials have extensively reported on the efficacy and safety of chronic treatment with phosphodiesterase type 5 inhibitors (PDE5-Is) - either alone or in combination with conventional therapies - in ameliorating LUTS in men with or without ED. ${ }^{2}$

The aims of the present review were to analyze the links between LUTS/BPH and

Correspondence: Mauro Gacci Department of Urology, University of Florence, Viale A Gramsci 7, 5012 I Florence, Italy

Tel +393396640070

Email maurogacci@yahoo.it

submit your manuscript | www.dovepress.com 


\section{The emerging links between LUTS and ED in aging men Epidemiologic data}

Many authors have analyzed the association between LUTS/ $\mathrm{BPH}$ and ED in aging men. ${ }^{3}$ The strong and independent relationship between LUTS and ED has been shown in several epidemiologic trials. The positive association of LUTS severity with clinically relevant ED - independent of wellknown causes of ED such as age, diabetes, medications, or coronary artery disease - has strongly suggested a common pathogenetic mechanism.

The National Health and Social Life Survey suggested that LUTS are a significant risk factor for ED, with an odds ratio (OR) of 3.13 in 1410 men aged $18-59$ years. ${ }^{4}$ In the Krimpen study, which included men aged 50-78 years, the prevalence of severe ED was tenfold higher in men aged 70-78 years than in those aged 50-54 years. Logistic regression showed that, regardless of age, severe LUTS were strongly associated with ED (OR 7.5; compared with no LUTS). ${ }^{5}$ The Asian Survey of Aging Males, a study on 1155 men aged 50-80 years, demonstrated that males with severe LUTS were three times more likely to have ED than those without LUTS (OR 3.17). ${ }^{6}$ In recent times, the Boston Area Community Health Survey investigated 5506 men aged 30-80 years and suggested that the relationship between LUTS and ED is primarily due to symptoms of prostatitis, incontinence, and nocturia (OR for ED: 1.86, 1.73, and 0.76 for prostatitis, incontinence, and nocturia respectively). ${ }^{7}$ In this study, only age $>60$ years, diabetes, and low socioeconomic status were more relevant than LUTS as predictive factors for ED.

In a study of 1420 men with LUTS, although age has been shown to be the most important predictor of sexual function, benign prostatic hyperplasia (BPH)-related symptoms (according to the Benign Prostatic Hyperplasia Impact Index [BII]), as well as general quality of life (QOL) were also predictive for sexual dysfunction, including ED. In contrast, uroflowmetry parameters or post-void residual (PVR) urine volume were not associated with sexual dysfunction. ${ }^{8}$ In a representative population sample of 4489 German men aged 30-80 years (Cologne Male Survey ${ }^{11}$ ), the overall prevalence of ED was $19.2 \%$, ranging from $2 \%$ in men in their 40 's to $53 \%$ in men in their 80 s; the prevalence of LUTS was $72.2 \%$ in men with ED, compared with $37.7 \%$ in those without ED. The Multinational Survey of the Aging Male (MSAM-7) investigated 12,815 men aged $50-80$ years and demonstrated an ED prevalence of $43.0 \%$, $65.8 \%$, and $82.5 \%$ in men with mild, moderate, and severe
LUTS, respectively; severe LUTS resulted in an OR of 7.67 for ED. ${ }^{9}$ The Epidemiology of LUTS survey of 11,834 men with a mean age of 56 years showed that men with LUTS had more severe ED; additionally, urgency with fear of urinary leakage and leakage during sexual activity were also positively associated with ED. ${ }^{10}$

In a recent comprehensive review that summarized data obtained in 20 community- and clinical-based studies of 71,322 men, a positive correlation between LUTS and ED was demonstrated, with the overall ORs ranging from 1.4 to $9.74 .^{11}$

Taken together, this evidence implies that both LUTS/ $\mathrm{BPH}$ and $\mathrm{ED}$ are associated with aging, but that the association between severity of LUTS/BPH and ED is independent of age. Alterations in mechanisms associated with metabolic syndrome and cardiovascular disorders are crucial to understanding the pathways and underlying links between these symptoms.

\section{Common pathways linking LUTS/BPH and ED}

The pathophysiology of LUTS/BPH and ED is complex and likely to be multifactorial involving numerous mechanisms affecting the entire lower urinary tract (LUT). The major mechanisms determining LUTS have been extensively reviewed elsewhere. ${ }^{12}$ Briefly, these are: reduced nitric oxide (NO)/cyclic guanosine monophosphate (cGMP) mainly in the prostate, urethra, and bladder but also in the pelvic neuronal and vascular bed; increased RhoA kinase pathway activity; increased autonomic nerve activity; and pelvic hypoxygenation and ischemia.

The altered balance between relaxation (reduced $\mathrm{NO} /$ cGMP-signaling) and contraction (increased RhoA/Rho kinase activity) in the penile smooth muscle compartment, as well as penile hypoxygenation/ischemia, are pathogenic factors underlying ED. Essentially, in animal models of ED associated with cardiovascular diseases - including hypertension, diabetes, and metabolic syndrome or neurogenic $\mathrm{ED}^{15-17}$ - the concomitant impairment of smooth muscle relaxation and reduced oxygenation were documented.

In humans, phosphodiesterase type 5 (PDE5) is expressed in the whole of the LUT, including the urethra, prostate, and bladder-all potential targets of PDE5-Is. ${ }^{13-16}$ In all these organs, PDE5 was prominently localized in the stroma and in the vascular bed (endothelial and smooth muscle cells), suggesting a possible action of PDE5-I either on smooth muscle contraction and/or blood flow. Animal models have provided a great deal of information on the possible mechanisms of action of PDE5Is, including the effects of tadalafil on LUT. Chronic pelvic 
ischemia was one of the main determinants of the functional and morphological changes observed in both bladder and prostate in the spontaneous hypertensive rat model. ${ }^{16,23}$

Chronic treatment with tadalafil or other PDE5-I in the spontaneous hypertensive rat was able to counteract all these LUT alterations, most likely through an increased blood perfusion and LUT oxygenation. ${ }^{15,16} \mathrm{~A}$ similar effect of tadalafil concerning increasing tissue perfusion and oxygenation has also been described in an animal model of severe neurogenic ED; chronic treatment with tadalafil after bilateral cavernous nerve neurotomy in the rat normalized penile oxygenation as well as smooth muscle content. One of the best described mechanisms of action of PDE5-Is with regard to inducing vasodilatation and increasing blood flow is smooth muscle cell relaxation of the LUT mediated by NO/cGMP. ${ }^{15,16}$ In addition, modulation of autonomic nervous system overactivity and bladder/prostate afferent nerve activity by PDE5-Is has also been suggested. ${ }^{12,19,20}$

Recently, it was documented that tadalafil enhances prostate and bladder neck relaxation through the inhibition of neurogenic contractions. ${ }^{18}$ Accordingly, it has been demonstrated that PDE5-Is induce a cGMP/protein kinase G-mediated inhibition of the contractile RhoA/Rhoassociated protein kinase signaling in the bladder, ${ }^{19}$ which also suggests a crucial role of PDE5-Is in ameliorating the dynamic/functional component of LUTS/BPH pathogenesis. These preclinical hypotheses have been confirmed by clinical evidence with PDE5-Is in spinal cord injured men investigated by urodynamics ${ }^{20}$ and in men under sexual rehabilitation for post-prostatectomy ED. ${ }^{21}$

A specific action of tadalafil in counteracting the pathogenic factors mainly related to the static component of LUTS (ie, prostate enlargement and urethral compression) has been demonstrated as well. ${ }^{18}$ We recently reported, for the first time, a specific action of tadalafil on the prostatic chronic inflammatory process, the third recognized pathogenetic component of LUTS/BPH. ${ }^{22,23,28}$

Although primarily characterized by increased proliferation of both fibroblastic stromal and epithelial cells, intraprostatic chronic inflammation has also been documented to play a causative role in LUTS/BPH. From a pathophysiological standpoint, an autocrine/paracrine proinflammatory loop between chronically activated $\mathrm{T}$ cells and stromal cells seems to be the key determinant factor in $\mathrm{BPH}$ development and progression. $^{24}$

The prostate of the adult male rabbit fed a high fat diet (HFD) developed severe histological inflammation coupled with stromal derangement and hypoxia. ${ }^{25-30}$
HFD-induced Metabolic Sydrome (MetS)-like features were associated with marked histological alterations of the prostate gland, characterized by severe inflammation coupled with stromal derangement and hypoxia. Interestingly, the HFD prostate showed also a marked increased expression of PDE5, suggesting that prostate in MetS condition could be the optimal target for PDE5-Is. PDE5 expression in the prostate was also strongly and positively associated with the expression of several inflammatory, myofibroblast activation, and hypoxia-related markers. ${ }^{28}$

Chronic use of a PDE5-I (12-week treatment with tadalafil) reduced the expression of inflammatory, pro-fibrotic, and myofibroblast activation markers in the prostate. ${ }^{28}$ Consistent with these data, it has previously been reported that tadalafil strongly attenuated tumor growth factor beta 1-induced fibroblast-tomyofibroblast trans-differentiation in primary human prostate stromal cells. ${ }^{31}$ Short-term treatment (1 week) with tadalafil was also able to significantly blunt HFD-related prostatic alterations, mainly reducing the inflammatory process. A significant reduction in interleukin 8, tumor necrosis factor alpha, and genes related to tissue remodeling was observed. ${ }^{28}$ Interleukin 8 is considered as a reliable surrogate marker of prostatic inflammatory diseases, ${ }^{32}$ linking chronic inflammation to prostatic enlargement. In particular, an increase in subtype-1 T-helper cells (Th1) is crucial in loss of self-tolerance and autoimmunetissue remodeling with hyperplastic overgrowth. Interestingly, we found that Th1 was absent from healthy rabbit prostate but was significantly induced by an HFD. ${ }^{23,27}$

Remarkably, both acute and chronic treatment with tadalafil dramatically reduced Th1 immune response in the prostates of animal subject on HFDs, further indicating the inhibitory action of PDE5-Is on the inflammatory processes affecting the prostate during metabolic derangements. Therefore, animal models demonstrated that both chronic and acute tadalafil administration were effective in reducing the HFD-related prostatic alterations, mainly attenuating chronic inflammatory and pro-fibrotic processes. ${ }^{28}$ Other positive effects of tadalafil on LUT oxygenation have also been reported. ${ }^{16}$

In conclusion, these experimental findings in animals and studies in humans add new insights into the understanding of the mechanism of action of PDE5-Is, and in particular of tadalafil, in alleviating LUTS (even in MetS patients). Study results strongly support the multiple potentiality of this drug class.

\section{Active treatments for LUTS/BPH and their impact on sexual function}

Active treatments include phytotherapy, conventional medical therapies, and surgical procedures 
(minimally invasive or invasive). ${ }^{33}$ The more invasive the treatment, the greater the occurrence of adverse events (AEs). Consequently, while invasive surgery (eg, transurethral resection of the prostate [TURP]) can sufficiently and quickly decrease symptoms or signs of BPH, it is also associated with well-defined severe AEs, such as bleeding, the need for blood transfusions, transurethral resection syndrome, urinary incontinence, and ED. Therefore, it is crucial that any treatment for LUTS/BPH relieves the LUTS without producing major or troublesome AEs. Both medical and surgical treatments can have problematic side effects, including a remarkable worsening of sexual function, but these particular AEs appear in different treatment modalities with different probabilities. ${ }^{1}$

The efficacy of the various treatments and any sexual dysfunction/ED side effects they might produce have been well documented for the various treatment options of LUTS/BPH.

\section{Phytotherapy}

The evidence of phytotherapy for LUTS/BPH has strikingly increased during the last two decades. Plant extracts contain a large variety of chemical components, including phytosterols, plant oils, fatty acids, and phytoestrogens, which have various biochemical effects - including anti-inflammatory, antioxidant, and inhibitory - on 5 $\alpha$-reductase. In the TransEuropean Research Into the Use of Management Policies for LUTS suggestive of BPH in Primary Healthcare (TRIUMPH) study, Serenoa repens and Pygeum africanum, the most used phytotherapeutic agents, both produced an equally significant improvement in LUTS in $43 \%$ of patients, giving a mean change of 3 International Prostate Symptom Score (IPSS) points but were less effective than $\alpha$-blockers or $5 \alpha$-reductase inhibitors (5ARIs). ${ }^{34}$ However, only mild and infrequent AEs, with particular concern regarding the impact on sexual function, (decreased libido) or gastrointestinal function, have been reported. In a trial with 811 LUTS/BPH patients, the incidence of ejaculatory dysfunction was reported to be significantly lower with Serenoa repens than with tamsulosin ( $0.6 \%$ vs $4.2 \%$ respectively). ${ }^{35}$ In another study on 2511 men, an increase in sexual dysfunction, evaluated using the Male Sexual Function-4 questionnaire, was reported with tamsulosin $(+0.3)$ and finasteride $(+0.8)$, while a slight improvement with Serenoa repens $(-0.2)$ was documented. ${ }^{36}$

\section{Medical therapies}

Medical therapies for LUTS/BPH mainly include $\alpha$-blockers and 5ARIs, alone or in combination, with well-established efficacy and AE profiles. ${ }^{1}$ In the TRIUMPH study, which recorded the treatment and outcomes of 2351 newly presenting LUTS/BPH patients in six European countries over 3 years, the use of $\alpha$-blockers alone resulted in the most effective monotherapy, with a mean reduction of 6.3 IPSS points. In contrast, 5ARIs reduced LUTS by only 4.1 IPSS points. However, a statistically significant advantage resulted when using the combination therapy of tamsulosin plus finasteride, which resulted in a decrease of 8.1 IPSS points. ${ }^{34}$

Despite the fact that some $\alpha$-blockers (eg, alfuzosin) improved EF in some trials, ejaculatory dysfunction was usually reported in these trials, occurring in 2\%-14\% of patients. ${ }^{37}$ Further, several sexual AEs have been reported to result from treatment with 5ARIs, including impaired sexual desire or loss of libido in $2 \%-10 \%$ of patients, ED in $3 \%-16 \%$, and ejaculatory dysfunction in $0 \%-8 \%{ }^{38}$ The Combination of Avodart and Tamsulosin (CombAT) study evaluated tamsulosin and dutasteride combination therapy in 4844 men with signs and symptoms of BPH; after 24 months, the rate of ED was $3.8 \%$ with tamsulosin, $6.0 \%$ with dutasteride, and $7.4 \%$ with combination therapy. ${ }^{39}$

\section{Minimally invasive procedures}

In recent years, a number of minimally invasive therapies for LUTS/BPH have been developed and refined, including transurethral needle ablation (TUNA), transurethral microwave thermotherapy, and interstitial laser coagulation. Although these procedures are less effective at relieving LUTS/ BPH than conventional surgical treatments (eg, TURP) - with a mean 10.2 and 9.1 IPSS point reduction at 1 year for transurethral microwave thermotherapy and TUNA, respectively ${ }^{40}-$ AEs such as bleeding, ED, or ejaculatory dysfunction occur at a lower frequency. Consequently, their attractiveness for both patients and physicians is increasing. In a systematic review and meta-analysis of TUNA in symptomatic BPH patients, ED was reported in only $0.3 \%$ of men, retrograde ejaculation in $0.2 \%$, and loss of ejaculation in $0.08 \%{ }^{41}$

\section{Conventional and new surgical treatments}

If treatment with drugs proves insufficient, the surgical gold standard for moderate to severe LUTS/BPH in patients with prostate volumes of less than $80-100 \mathrm{~mL}$ is TURP, which has replaced open prostatectomy $(\mathrm{OP}) .{ }^{42}$ Alternatively, for men with a prostate volume of $\leq 30 \mathrm{~mL}$, transurethral incision of the prostate or bladder neck incision may be undertaken. A retrospective meta-analysis of 3304 men treated by conventional surgical therapies reported retrograde ejaculation in $6 \%-80 \%$ of patients after OP, $70 \%$ after TURP, and $39 \%$ after transurethral incision of the prostate. ED was reported 
in $13 \%$ of patients after TURP and in $11 \%$ after $\mathrm{OP}^{43}$ The newest treatments for signs or symptoms of BPH are holmium laser enucleation of the prostate and photoselective vaporization of the prostate; fewer AEs (eg, bleeding or blood transfusions) have been reported for both procedures than for conventional surgery, but their impact on sexual function was almost the same. ${ }^{44}$

Although the efficacy and AE profiles of all currently available treatment options for LUTS/BPH have been well investigated and defined, outcomes are sometimes unsatisfactory for patients and partners, mainly due to the postoperative AEs - above all, those affecting sexual function. These treatment-related disadvantages have triggered the search for new treatment options for effective relief of LUTS that result in very few or no sexual or other AEs. PDE5-I treatment (eg, with tadalafil [Cialis ${ }^{\mathrm{TM}}$, Eli-Lilly, Indianapolis, IN, USA]) seems to combine the efficacy of drugs ( $\alpha$-blockers) and can maintain or even improve sexual function.

\section{Clinical evidence for tadalafil for LUTS/BPH}

In 2007, McVary et $\mathrm{a}^{45}$ evaluated for the first time the efficacy and safety of tadalafil for the treatment of LUTS/BPH in men with or without ED. A total of 479 patients were screened and, after a 4-week washout and 4-week placebo run-in period, 281 were randomly assigned to a 6 -week treatment with once-daily placebo or tadalafil $5 \mathrm{mg}$. After 6 weeks, the remaining 261 patients were assigned to continue with placebo for another 6 weeks (a total of 12 weeks of once-daily placebo treatment) or to dose escalate tadalafil to $20 \mathrm{mg}$ once daily. Of 143 placebo-assigned patients, $121(84.6 \%)$ were sexually active, $84(59.2 \%)$ had no ED, and $76(53.1 \%)$ were sexually active despite ED, while, of 138 men treated with 5 or $20 \mathrm{mg}$ tadalafil, 107 (77.5\%) were sexually active, 99 (71.7\%) had normal sexual function, and $80(58.0 \%)$ were sexually active despite ED. Patients were evaluated for LUTS using the IPSS (including the IPSS-QOL question) and BII questionnaires. Maximum urinary flow rate $\left(\mathrm{Q}_{\max }\right)$ and average urinary flow rate $\left(\mathrm{Q}_{\mathrm{ave}}\right)$ of free uroflowmetry were recorded, post-void residual urine (PVR) was measured by ultrasound after uroflowmetry, and sexual function was studied by using the erectile function (EF) domain of the International Index of Erectile Function (IIEF) questionnaire (questions 1 to 5 and 15).

In McVary et al's study, ${ }^{45}$ tadalafil significantly improved LUTS at week 6 (mean IPSS change from baseline for $5 \mathrm{mg}$ tadalafil was -2.8 compared with -1.2 for placebo) but improvement of LUTS was even better at week 12 with dose escalated tadalafil $(5 / 20 \mathrm{mg}$ tadalafil -3.8 vs placebo -1.7$)$.
BII changes observed were -0.7 for tadalafil $5 \mathrm{mg}$ versus -0.4 for placebo at week 6 and -1.3 for tadalafil versus -0.6 for placebo at week 12 . No significant changes of uroflowmetry parameters were observed after week 12 between placebo and tadalafil: $\mathrm{Q}_{\max } 2.3 \mathrm{~mL} / \mathrm{s}$ versus $2.7 \mathrm{~mL} / \mathrm{s}(P=0.81)$; $\mathrm{Q}_{\text {ave }} 1.1 \mathrm{~mL} / \mathrm{s}$ versus $0.9 \mathrm{~mL} / \mathrm{s}(P=0.79)$. Additionally, there was no change of PVR between the groups: $-1.2 \mathrm{~mL}$ versus $-2.5 \mathrm{~mL}(P=0.57)$. As expected, sexual activity, as measured by the IIEF questionnaire, significantly improved only with tadalafil $(+8.4)$ compared with placebo $(+1.6$; $P<0.001)$ after week 12. Also in this study, the authors were the first to evaluate the safety profile for PDE5-Is in patients with LUTS/BPH. The most commonly reported AEs $(>2 \%)$ were dyspepsia, back pain, headache, nasopharyngitis, and upper respiratory tract infection (each AE occurred in $\leq 5.1 \%$ ). As expected, erections improved after 12 weeks with $5 / 20 \mathrm{mg}$ tadalafil in 5.1\%. Thus, in this study, McVary et al demonstrated for the first time that tadalafil is an effective and safe treatment for LUTS/BPH; patients achieved an effective LUTS response without significant AEs but with improvement of concomitant ED.

In 2008, Roehrborn et $\mathrm{al}^{46}$ published their report on a randomized, double-blind, placebo-controlled, 12-week study performed in ten countries and 92 centers in order to evaluate the optimal dose of tadalafil for the treatment of LUTS/ BPH. The authors included 1813 LUTS/BPH patients aged 45 years or older. After a 4-week washout and 4-week placebo run-in period, the study population was equally divided into five treatment arms: either (1) placebo or tadalafil (2) $2.5 \mathrm{mg}$, (3) $5 \mathrm{mg}$, (4) $10 \mathrm{mg}$, or (5) $20 \mathrm{mg}$ once daily. Patients were assessed at baseline and at weeks 4, 8, and 12. At week 12, patients' IPSS had significantly improved for all tadalafil doses compared with placebo. However, the best IPSS/QOL/ $\mathrm{AE}$ ratios were obtained with tadalafil $5 \mathrm{mg}$. Compared with placebo (decrease of 2.3 IPSS points), improvement of IPSS was 3.9 for tadalafil $2.5 \mathrm{mg}(P<0.015), 4.9$ for tadalafil $5 \mathrm{mg}(P<0.001), 5.2$ for tadalafil $10 \mathrm{mg}(P<0.001)$, and 5.2 for tadalafil $20 \mathrm{mg}(P<0.001)$. In terms of safety profile, back pain, myalgia, and headache were more frequent at higher tadalafil doses. AEs reported in the placebo arm versus 2.5/5/10/20 mg tadalafil arms were: six (2.8\%) versus five, six $(2.8 \%)$, eleven $(5.1 \%)$, and seven $(3.3 \%)$ for headache; zero versus two (1.0\%), ten (4.7), six (2.8\%), and ten $(4.8 \%)$ for dyspepsia; and one $(0.5 \%)$ versus three $(1.4 \%)$, two $(0.9 \%)$, ten $(4.6 \%)$, and twelve $(5.7 \%)$ for back pain. AEs led to study discontinuation for $2.4 \%$ of placebotreated men versus $1.9 \%, 5.7 \%, 5.1 \%$, and $6.7 \%$, respectively, of 2.5/5/10/20 mg tadalafil-treated males. Thus, this study 
determined that tadalafil $5 \mathrm{mg}$ once daily is the best dosage for the treatment of LUTS/BPH, providing an effective response to LUTS, with a minimal occurrence of AEs and rate of discontinuation.

Also in 2008, Bechara et $\mathrm{al}^{47}$ considered combination therapy of tadalafil with an $\alpha$-blocker for the treatment of LUTS/BPH for the first time and compared tamsulosin $0.4 \mathrm{mg}$ with tamsulosin $0.4 \mathrm{mg}$ plus tadalafil $20 \mathrm{mg}$ once daily. They enrolled 30 men with LUTS/BPH of at least 6 months in a randomized, double-blind, crossover study. Patients were treated with monotherapy or combination therapy for 45 days and then switched to the other treatment mode for another 45 days. The authors found a significant improvement in IPSS (tamsulosin -6.7 vs tadalafil/tamsulosin $-9.2 ; P<0.05$ ) and for the QOL question in the IPSS questionnaire (IPSS-QOL) $(P<0.001)$ with both treatment modalities compared with baseline but differences were greater with combination therapy. In terms of $\mathrm{Q}_{\max }$ and PVR, there was a significant improvement from baseline with both treatment regimens $(P<0.001)$ but no significant differences between tamsulosin alone versus tamsulosin plus tadalafil $20 \mathrm{mg}(P>0.05)$. Improvement in IIEF score was significant with tamsulosin plus tadalafil $(P<0.001)$ but not with tamsulosin alone $(P>0.05)$. In this study, therefore, the authors demonstrated for the very first time that combination therapy is more efficacious when adding a PDE5-I (tadalafil) to standard treatment (tamsulosin) than $\alpha$-blocker monotherapy.

In 2009, Liguori et $\mathrm{al}^{48}$ considered the combination of tadalafil $20 \mathrm{mg}$ with alfuzosin $10 \mathrm{mg}$ for the treatment of LUTS/BPH and ED. The authors enrolled 66 men who were randomized to either alfuzosin $10 \mathrm{mg}$ once daily (22 patients), tadalafil $20 \mathrm{mg}$ on alternate days (21 patients), or a combination of both ( 23 patients) and assessed treatment outcomes at baseline and at week 12 . They did not find a significant IPSS improvement for tadalafil alone (-8.4\%, $P$ not significant) but demonstrated a significant reduction in IPSS for alfuzosin monotherapy $(-27.2 \%$, $P<0.003)$ and combination therapy $(-41.6 \%, P<0.001)$. As such, Liguori et al confirmed the improved efficacy of improving LUTS/BPH (according to IPSS) with combination therapy ( $\alpha$-blockers plus a PDE5-I) compared with monotherapy ( $\alpha$-blocker or PDE5-I). Furthermore, the authors found an increased efficacy for EF and uroflowmetry parameters when using the combination therapy: IIEF $+37.6 \%(P<0.001)$ and $\mathrm{Q}_{\max }+29.6 \%(P<0.001)$ versus $\alpha$-blocker monotherapy (IIEF $+15.0 \%, P=0.026 ; \mathrm{Q}_{\max }$ $+21.7 \%, P=0.006$ ) or tadalafil monotherapy (IIEF $+36.3 \%$, $\left.P=0.011 ; \mathrm{Q}_{\max }+9.5 \%, P=0.044\right)$. At the end of the trial,
15 AEs (55.5\%) occurred in the combination therapy arm and five $(18.5 \%)$ in the tamsulosin/placebo arm; headache was the most reported AE: two patients discontinued the study due to AEs. This trial confirmed the efficacy and safety of combination therapy, even when using another $\alpha$-blocker (alfuzosin) compared with $\alpha$-blocker monotherapy.

In 2009, Porst et $\mathrm{al}^{49}$ investigated a selected group of 581 men with comorbid LUTS/BPH and ED who reported being sexually active. Mean age was 62 years and mean body mass index (BMI) was $28.3 \mathrm{~kg} / \mathrm{m}^{2}$. The majority of patients $(84.5 \%)$ had had LUTS/BPH for more than 1 year, and $50.4 \%$ had a LUTS/BPH history $>3$ years. After 4 -week washout period for patients taking BPH and/or ED drugs and 4-week placebo run-in period, patients were assigned to once-daily placebo or tadalafil $2.5,5,10$, or $20 \mathrm{mg}$ for 12 weeks. At baseline, the majority of patients had moderate ED (IIEF score of between 11 and 16): 60.0\% in the placebo group, $57.5 \%$ in the tadalafil $2.5 \mathrm{mg}$ group, $57.3 \%$ in the tadalafil $5 \mathrm{mg}$ group, $48.3 \%$ in the tadalafil $10 \mathrm{mg}$ group, and $57.8 \%$ in the tadalafil $20 \mathrm{mg}$ group. Improvement in IPSS from baseline to study end was significantly greater for all tadalafil doses versus placebo: -2.1 for placebo, -3.6 for tadalafil $2.5 \mathrm{mg}(P=0.043),-4.2$ for tadalafil $5 \mathrm{mg}$ $(P=0.004),-4.7$ for tadalafil $10 \mathrm{mg}(P<0.001)$, and -4.7 for tadalafil $20 \mathrm{mg}(P=<0.001) . \mathrm{Q}_{\max }$ was not significantly different for any tadalafil group versus placebo. In terms of sexual function in men with an IIEF score $<26$ at baseline, normal EF (IIEF $\geq 26$ ) was dose-dependently obtained for tadalafil-treated men: $21.2 \%$ with tadalafil $2.5 \mathrm{mg}, 34.2 \%$ with tadalafil $5 \mathrm{mg}, 42.5 \%$ with tadalafil $10 \mathrm{mg}$, and $40 \%$ for tadalafil $20 \mathrm{mg}$ versus $14 \%$ for placebo-treated men. AEs occurring in $\geq 2 \%$ of tadalafil-treated patients were headache, dyspepsia, back pain, and myalgia. For the $2.5,5,10$, and $20 \mathrm{mg}$ tadalafil groups, the discontinuation frequency due to AEs was $2.7 \%, 5.1 \%, 5 \%$, and $5.2 \%$, respectively. Thus, this dose-finding study confirmed the efficacy of tadalafil $5 \mathrm{mg}$ once daily in ameliorating LUTS/BPH and corroborated the role of this daily treatment in the concomitant improvement of EF in LUTS/BPH men with or without ED.

Also in 2009, for the first time, Roehrborn et al ${ }^{50}$ specifically evaluated uroflowmetry data in LUTS/BPH patients treated with tadalafil once daily. After a 4-week placebo run-in period, they enrolled 1058 LUTS/BPH patients and randomly allocated these men to receive once-daily treatment with placebo or tadalafil $(2.5,5,10$, or $20 \mathrm{mg})$ for 12 weeks. Uroflowmetry variables were stratified by age, $Q_{\max }$, LUTS severity, prostate size, previous $\alpha$-blocker use, and sexual activity, including ED history. The authors demonstrated that 
tadalafil was able to induce a dose-dependent increase in $\mathrm{Q}_{\max }$, although not one that was significantly different from placebo $(1.4 \mathrm{~mL} / \mathrm{s}$ [+15\%] for tadalafil $2.5 \mathrm{mg}, 2.0 \mathrm{~mL} / \mathrm{s}$ [+22\%] for tadalafil $20 \mathrm{mg}$, and $1.2 \mathrm{~mL} / \mathrm{s}$ [+12\%] for placebo). Moreover, age, $\mathrm{Q}_{\max }$ severity at baseline, ED history, sexual activity, and previous treatment with $\alpha$-blockers were determinants for the change in $\mathrm{Q}_{\max }$ across all tadalafil and placebo treatment groups. In tadalafil $2.5,5,10$, or $20 \mathrm{mg}$ once daily groups, there were study discontinuations due to AEs in $1.9 \%, 5.7 \%$, $5,1 \%$ and $6.7 \%$, respectively. Once again, the lack of a significant $\mathrm{Q}_{\max }$ improvement was documented in this trial; this finding has since been confirmed by sub-analyses of other tadalafil trials. $^{2}$

In 2010, Dmochowski et $\mathrm{al}^{51}$ conducted a multicenter, randomized, double-blind, placebo-controlled, 12-week trial comparing once-daily tadalafil $20 \mathrm{mg}$ with placebo in LUTS/ $\mathrm{BPH}$ men with or without bladder outlet obstruction. Invasive computer-urodynamic evaluation, free uroflowmetry, and IPSS were assessed in this study. However, men with severe bladder outlet obstruction were not included in this study. In 101 placebo-treated patients (mean age 59 years and BMI $\left.29.4 \mathrm{~kg} / \mathrm{m}^{2}\right)$, mean $\mathrm{Q}_{\max }$ was $13.3 \pm 7.4 \mathrm{~mL} / \mathrm{s}$ and mean PVR $59.3 \pm 60.9 \mathrm{~mL}$, while the 99 tadalafil-treated patients (mean age 58.2 years and BMI $29.5 \mathrm{~kg} / \mathrm{m}^{2}$ ) had a mean $\mathrm{Q}_{\max }$ of $15.4 \pm 11.1 \mathrm{~mL} / \mathrm{s}$ and a mean PVR of $45.7 \pm 49.6 \mathrm{~mL}$. The differences of $Q_{\max }$ and $Q_{\text {ave }}$ between men treated with tadalafil $20 \mathrm{mg}$ versus placebo were not statistically significant for free uroflowmetry (mean difference of change from baseline tadalafil vs placebo: $\mathrm{Q}_{\max }-0.6 \mathrm{~mL} / \mathrm{s}$ and $\mathrm{Q}_{\text {ave }}+1 \mathrm{~mL} / \mathrm{s}$ ) or pressure flow study $\left(\mathrm{Q}_{\max }-0.1 \mathrm{~mL} / \mathrm{s}\right.$ and $\left.\mathrm{Q}_{\mathrm{ave}}+0.1 \mathrm{~mL} / \mathrm{s}\right)$. The incidence of AEs was higher in the tadalafil group (55.6\%) than in the placebo group $(27.7 \%)$, but discontinuations due to AEs were low in both groups (tadalafil $2.0 \%$ vs placebo $1.0 \%$ ). In this safety study using a high dosage of tadalafil (20 mg for 12 weeks), Dmochowski et al suggested that once-daily administration of tadalafil should not worsen urodynamic parameters in men with LUTS/BPH.

In 2011, Porst et $\mathrm{al}^{52}$ evaluated the efficacy and safety of once-daily tadalafil $5 \mathrm{mg}$ for the treatment of LUTS/BPH in an international, randomized, double-blind, placebocontrolled, 12-week trial. The authors screened 442 men and, after 4-week washout period and 4-week placebo run-in period, randomized 325 patients to receive placebo or tadalafil $5 \mathrm{mg}$ once daily. From baseline to endpoint, tadalafil significantly improved IPSS in comparison to placebo ( $-5.6 \mathrm{vs}-3.6$, $P=0.004)$. There was a significant reduction in IPSS already at week 4 (tadalafil $5 \mathrm{mg}-5.3$ vs placebo $-3.5, P=0.003$ ) and after week 12 . Likewise, the BII score had already sig- nificantly improved at week 4 (tadalafil $5 \mathrm{mg}-1.8$ vs placebo $-1.2, P=0.029)$ and continued to improve until week 12 (tadalafil $5 \mathrm{mg}-1.8$ vs placebo $-1.3, P=0.057$ ). As in other studies, Porst et al found a remarkable improvement in IIEF in sexually active men with ED who were treated with tadalafil $5 \mathrm{mg}$ versus placebo (+6.7 vs $+2.0, P<0.001)$ at week 12 . The incidence rate of at least one AE was $22 \%$ and $26.1 \%$, and one $(0.6 \%)$ and six $(3.7 \%)$ patients discontinued due to AEs in the placebo and tadalafil $5 \mathrm{mg}$ groups, respectively. As such, this study demonstrated that the clinical efficacy of tadalafil $5 \mathrm{mg}$ once daily in treatment of LUTS/BPH is already detectable after 4 weeks.

In 2011, Donatucci et $\mathrm{al}^{53}$ evaluated the long-term efficacy and safety of tadalafil for LUTS/BPH. They enrolled 427 men who had been investigated in a 12-week, placebocontrolled, dose-finding study with once-daily tadalafil (2.5, 5,10 , or $20 \mathrm{mg}$ ) or placebo ${ }^{46}$ and continued treatment in the open-label extension study with tadalafil $5 \mathrm{mg}$ for 52 weeks. Independent of the dosage used in the 12-week dose-finding period, at endpoint, there was an improvement of $-5.0 \pm 6.7$ points in IPSS and of $5.9 \pm 7.6$ points in IIEF score. After 1 year, compared with at week 12 , a further improvement in total IPSS and storage and voiding sub-scores was reported $(-0.9 \pm 5.7,-0.3 \pm 2.6$, and $-0.6 \pm 3.6$, respectively). The most common AEs ( $\geq 2 \%$ ) were dyspepsia, gastroesophageal reflux disease, back pain, headache, sinusitis, hypertension, and cough. A total of 22 men (5.2\%) discontinued the study due to AEs. Thus, this long-term study showed that tadalafil $5 \mathrm{mg}$ once daily is well tolerated during a treatment period of 1 year and that the drug maintains or even improves storage and voiding symptoms, but the cost-effectiveness of the long-term chronic use of tadalafil was not evaluated.

In 2012, Oelke et $\mathrm{al}^{54}$ evaluated for the first time tadalafil $5 \mathrm{mg}$ and tamsulosin $0.4 \mathrm{mg}$ once daily for the treatment of LUTS/BPH in an international, placebo-controlled, randomized, double-blind, 12-week trial comparing separately and independently these two drugs with placebo. Following screening and washout, if needed, and a 4-week placebo run-in, they evaluated 511 men who were randomly assigned to placebo $(n=172)$, tadalafil $5 \mathrm{mg}(\mathrm{n}=171)$, or tamsulosin $0.4 \mathrm{mg}(\mathrm{n}=168)$. Compared with placebo, there was a significant decrease in IPSS of 1.5 points after only 1 week of treatment with tadalafil or tamsulosin $(P<0.01)$. After 12 weeks, decrease in IPSS with tadalafil (2.1 points, $P=0.001$ ) was even more pronounced than with tamsulosin $(1.5, P=0.023)$ compared with placebo. Additionally, BII score had significantly improved at week 4 with tadalafil $(-0.8 ; P<0.001)$ and tamsulosin $(-0.9 ; P<0.001)$ and at week 12 (tadalafil $-0.8, P=0.003$; tamsulosin -0.6 , 
$P=0.026)$ compared with placebo. QOL (IPSS-QOL), treatment satisfaction (Treatment Satisfaction Scale), and Patient or Physician Global Impression of Improvement scores only improved for tadalafil, not for placebo or tamsulosin. Improvement in IIEF score was once more documented for tadalafil (4.0, $P<0.001)$ but not for tamsulosin $(-0.4, P=0.699)$ compared with placebo. The patient discontinuation rate due to AEs was $1.2 \%$ for placebo, $1.2 \%$ for tadalafil $5 \mathrm{mg}$, and $0.6 \%$ for tamsulosin $0.4 \mathrm{mg}$. The unexpected outcome of this study was the significant improvement of uroflowmetry parameters. $\mathrm{Q}_{\max }$ and $\mathrm{Q}_{\text {ave }}$ similarly increased with both tadalafil $(2.4 \mathrm{~mL} / \mathrm{s}$, $P=0.009$ and $1.6 \mathrm{~mL} / \mathrm{s}, P=0.002$, respectively) and tamsulosin $(2.2 \mathrm{~mL} / \mathrm{s}, P=0.014$ and $1.3 \mathrm{~mL} / \mathrm{s}, P=0.023$, respectively) at week 12 . In this trial, therefore, Oelke et $\mathrm{al}^{54}$ demonstrated a significant and clinically meaningful improvement in LUTS/ $\mathrm{BPH}$ with tadalafil $5 \mathrm{mg}$ once daily, which was similar to that attained by tamsulosin $0.4 \mathrm{mg}$ once daily after only 1 week, a further improvement in LUTS/BPH after 12 weeks, improvement of QOL and treatment satisfaction parameters only with tadalafil, and an unexpected but remarkable improvement of urinary flow rates at week 12 .

Also in 2012, Regadas et al ${ }^{55}$ evaluated for the first time the effects on free uroflowmetry of treating patients with LUTS/BPH for 30 days with tamsulosin $0.4 \mathrm{mg}$ with or without tadalafil $5 \mathrm{mg}$ in a cohort of 40 men with bladder outlet obstruction (mean \pm standard deviation: $\mathrm{Q}_{\max } 6.2 \mathrm{~mL} / \mathrm{s} \pm 2.6$ for $\alpha$-blockers alone and $7.4 \mathrm{~mL} / \mathrm{s} \pm 2.6$ for an $\alpha$-blocker plus tadalafil). Patients were equally randomized into two treatment arms: once-daily tamsulosin $0.4 \mathrm{mg}$ plus tadalafil $5 \mathrm{mg}$ or tamsulosin $0.4 \mathrm{mg}$ plus placebo. The study design included an urodynamic study based on the recommendations of the International Continence Society. Detrusor pressure at maximum flow was significantly reduced with tamsulosin plus tadalafil compared with tamsulosin alone $(13 \pm 17.0$ vs $-1.2 \pm 14.35$, $P=0.03)$. Moreover, $\mathrm{Q}_{\max }$ increased in both treatment groups $(1.0 \pm 2.4 \mathrm{~mL} / \mathrm{s}$ with tamsulosin + tadalafil and $1.4 \pm 2.4 \mathrm{~mL} / \mathrm{s}$ with tamsulosin + placebo), but differences were not significantly different $(P=0.65)$. Finally, LUTS/BPH decreased significantly in both the tadalafil plus tamsulosin and tamsulosin plus placebo groups ( -9.75 vs -6.0 IPSS points, $P=0.01$ ). No significant AEs led to discontinuation in the tadalafil plus tamsulosin group. This is the first trial - based on a computerurodynamic study - to have demonstrated that tadalafil reduces detrusor pressure at maximum flow and improves LUTS/BPH without significantly changing $\mathrm{Q}_{\max }$.

Again in 2012, Yokoyama et al ${ }^{56}$ directly compared tadalafil with tamsulosin $0.2 \mathrm{mg}$ in the first study to do so. The authors enrolled 612 men aged $\geq 45$ years who had a history of LUTS/BPH for at least 6 months, a total IPSS $\geq 13, \mathrm{Q}_{\max } 4-15 \mathrm{~mL} / \mathrm{s}$, and a prostate volume $>20 \mathrm{~mL}$. After a 2-week washout period and 4-week placebo leadin period, they randomized patients into four groups and treated patients with once-daily placebo, tadalafil $2.5 \mathrm{mg}$, tadalafil $5 \mathrm{mg}$, or tamsulosin $0.2 \mathrm{mg}$ for 12 weeks. From baseline to endpoint, LUTS/BPH significantly decreased by 4.8 IPSS points with tadalafil $2.5 \mathrm{mg}(P=0.003)$, 4.7 points with tadalafil $5 \mathrm{mg}(P=0.004)$, and 5.5 points with tamsulosin $0.2 \mathrm{mg}$ compared with 3.0 IPSS points with placebo. They observed that a relevant decrease in total IPSS $(P<0.05)$ was reached after 2 weeks of once-daily tadalafil $5 \mathrm{mg}$ treatment but only after 8 weeks for tadalafil $2.5 \mathrm{mg}$ treatment. $\mathrm{Q}_{\max }$ improvements were not significant with once-daily tadalafil or tamsulosin in this study. In terms of safety profile, the rate of discontinuation from each group due to AEs was $0.6 \%$ from the placebo group, $3.3 \%$ from the tadalafil $2.5 \mathrm{mg}$ group, $4.5 \%$ from the tadalafil $5.0 \mathrm{mg}$ group, and $1.3 \%$ from the tamsulosin group. In this study the authors demonstrated that tadalafil did not significantly modify prostate-specific antigen concentration during the 12-week treatment period (baseline to 12 weeks: 1.7 vs $1.8 \mathrm{ng} / \mathrm{mL}, P=0.083)$.

\section{Safety/tolerability and efficacy of tadalafil in systematic reviews and meta-analyses of men with LUTS/BPH Safety}

All PDE5-Is included in the systematic review authored by Liu et $\mathrm{al}^{57}$ showed a good safety profile with a relative risk of AEs from tadalafil similar to those reported with vardenafil or sildenafil (2.27 vs 1.86 vs 1.22, respectively). Moreover, the occurrence of serious AEs was rare for all PDE5-Is (1.1\%, $1.85 \%$, and $1.05 \%$ for the tadalafil, vardenafil, and sildenafil subgroups, respectively).

Martínez-Salamanca et $\mathrm{l}^{58}$ confirmed that tadalafil has a favorable tolerability profile, with most of the AEs being mild to moderate in severity. Moreover, discontinuation rates due to AEs were very low for tadalafil and comparable to those reported for placebo $(2.0 \%$ vs $1.0 \%)$. Headache, dyspepsia, back pain, gastroesophageal reflux, sinusitis, and myalgia were the most frequently reported AEs in clinical trials (11.1\%, 10.2\%, 11.1\%, 3.0\%, 0.3\%, and 5.7\%, respectively; Table 1). These same AEs were reported in the previously published PDE5-I meta-analysis, however, the probability of an $\mathrm{AE}$ was higher in the latter report. ${ }^{2}$ The overall incidence 


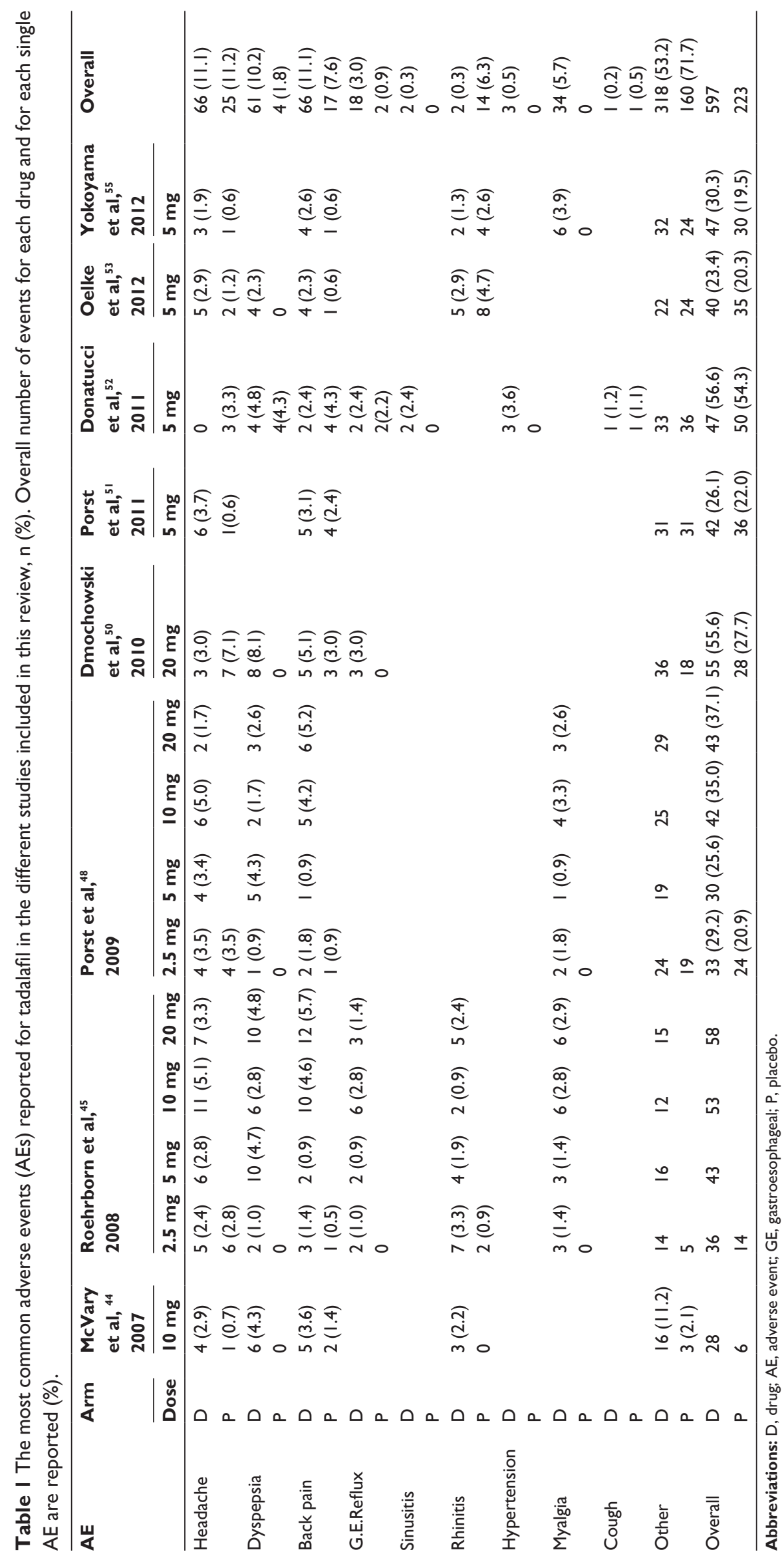


of AEs reported in the present review on tadalafil versus placebo was very similar to that reported by Gacci et $\mathrm{al}^{2}$ in the systematic review of all PDE5-Is versus placebo: $16.0 \%$ versus $6.0 \%$.

\section{Efficacy}

Liu et $\mathrm{al}^{57}$ included in their systematic review three studies (six RCTs) of tadalafil versus placebo, demonstrating a mean difference in IPSS change from baseline of 2.57 (95\% confidence interval [CI] 3.15-1.98) and in IIEF-EF score of 5.34 (95\% CI 4.11-6.57) in favor of tadalafil. In the same review, the meta-analysis of mean difference in $\mathrm{Q}_{\max }$ and $\mathrm{PVR}$ were not statistically significant: 0.20 (95\% CI 0.24-0.64, $P=0.38)$ and 0.47 (95\% CI 5.17-6.10, $P=0.87)$, respectively. In this review, the authors emphasized that there is no significant correlation between LUTS/BPH improvement (change in IPSS) and recovery of sexual activity (change in IIEF-EF score) and that the efficacy of tadalafil on LUTS/ $\mathrm{BPH}$ in men without ED demonstrates a direct activity of tadalafil on the LUT; in particular, the overall improvement of IPSS in men with ED overlapped the improvement in IPSS in men without ED (IPSS mean difference -2.57 vs -2.60 , respectively).

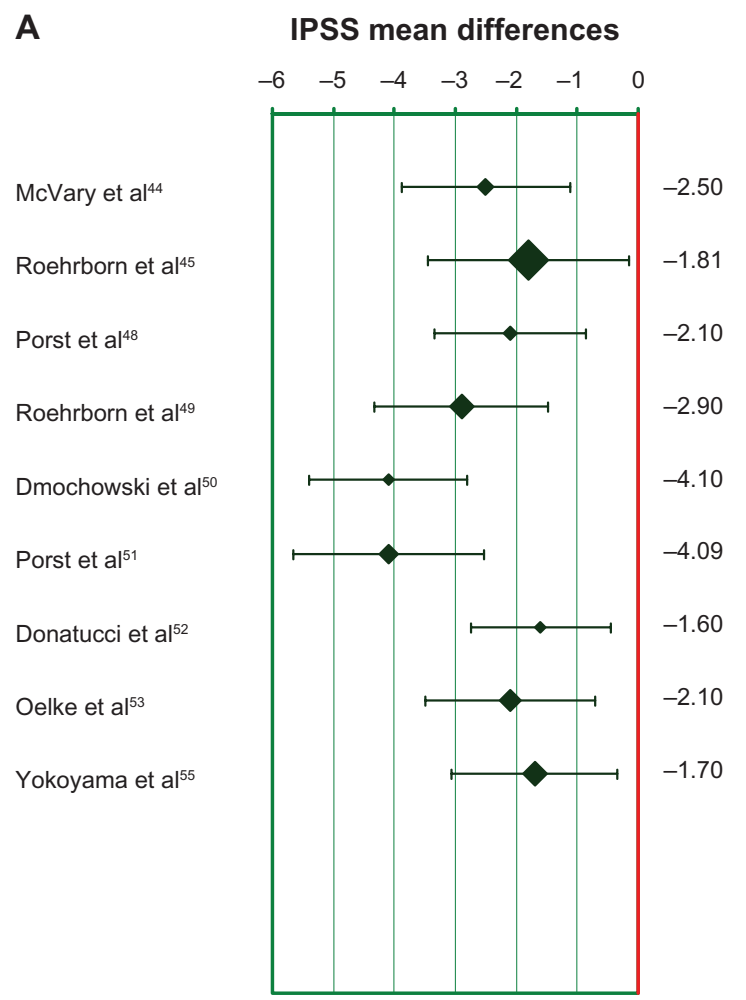

In 2011, in a nonsystematic descriptive review including data from 1088 men treated with tadalafil compared with 444 treated with placebo, Martínez-Salamanca et al $^{58}$ confirmed that tadalafil significantly improves LUTS/BPH compared with placebo (mean IPSS difference between treatments +4.2 , $P<0.001)$. The overall decrease in IPSS was comparable with that reported in previous $\alpha$-blocker studies. In the subgroup of sexually active men (55\%), a significant improvement in EF was also reported (IIEF-EF domain scores +7.15 with tadalafil vs +2.38 with placebo, $P=0.001$ ). Finally, none of the studies included in this review showed a significant effect of tadalafil on uroflowmetry variables.

The latest systematic review with meta-analysis by Gacci et $\mathrm{al}^{2}$ included four RCTs on tadalafil versus placebo (1360 vs 568 patients) and two RCTs on tadalafil plus $\alpha$-blocker versus $\alpha$-blocker alone (34 vs 32 patients). The overall outcomes in terms of efficacy as measured by IPSS, IIEF, and $\mathrm{Q}_{\max }$ were very similar to those reported for other PDE5-Is selected for the review (Figure 1). In particular, the most remarkable improvement in LUTS/BPH with tadalafil alone was obtained by Roehrborn et al's study ${ }^{46}$ (mean difference in change from baseline compared with placebo: IPSS -3.7, CI -5.5 to -1.8), while the maximum improvement in LUTS/BPH achieved tadalafil and $\alpha$-blocker combined

$\mathbf{B}$

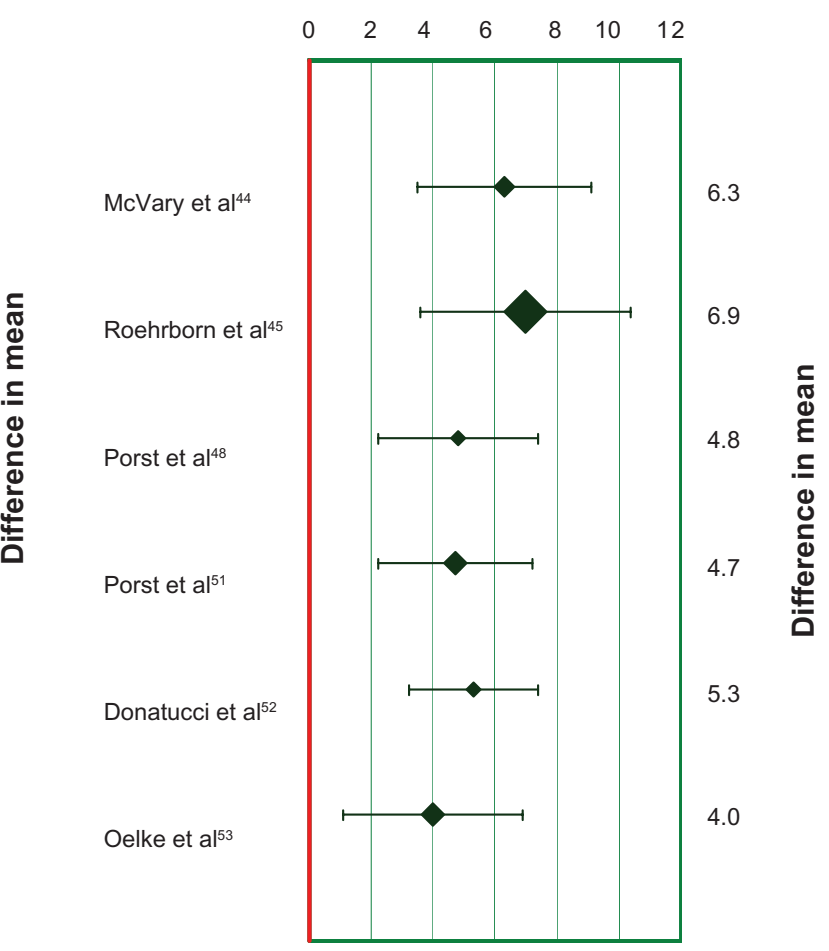

Figure I Weighted mean differences (with 95\% confidence interval) of International Prostate Symptom Score (IPSS) (A) and International Index of Erectile Function-Erectile Function (IIEF-EF) score (B) for the studies on tadalafil versus placebo. 
therapy compared with $\alpha$-blocker alone was reported by Bechara et $\mathrm{al}^{47}$ (IPSS -2.5, CI -5.7 to +0.7). Excellent results were obtained either with tadalafil alone or in combination with $\alpha$-blockers with regard to EF (IIEF +6.8, CI 4.3-9.2 and IIEF +6.3 , CI 0.9-11.7, respectively)..$^{45,47}$ Combination therapy with tadalafil and an $\alpha$-blocker achieved a statistically significant - albeit clinically negligible - improvement of $Q_{\max }$ (+0.9 and $+1.0 \mathrm{~mL} / \mathrm{s}$ respectively). ${ }^{47,48}$ Moreover, Gacci et al's ${ }^{2}$ meta-analysis demonstrated that younger men with lower BMI and more severe LUTS/BPH are the best candidates (target patients) for PDE5-I treatment when improvement of LUTS/ $\mathrm{BPH}$ is intended.

\section{Conclusion}

Many preclinical and clinical studies emphasize the close link between LUTS/BPH and ED. The majority of active treatments for LUTS/BPH have a negative impact on EF.

The remarkable improvement of both LUTS/BPH and ED with tadalafil $5 \mathrm{mg}$ once daily suggests a leading role for this PDE5-I treatment for men with comorbid BPH and ED. PDE5-I treatment is associated with a low rate of AEs and any AEs that do occur tend to be of low severity.

Further studies are needed to better understand the mechanisms of action of PDE5 or PDE5-Is, analyze the correct time for treatment, the cost-effectiveness of treatment, and to better define the long-term safety and efficacy of once-daily use of tadalafil $5 \mathrm{mg}$ for men with LUTS/BPH with or without ED.

\section{Disclosure}

Matthias Oelke has been advisor, lecturer, or trial investigator for Eli Lilly and Company as well as for Pfizer. Mario Maggi is a scientific consultant for Bayer Pharma AG, Germany and Eli-Lilly Indianapolis, Indiana Other authors declare no conflicts of interest in this work.

\section{References}

1. Gacci M, Eardley I, Giuliano F, et al. Critical analysis of the relationship between sexual dysfunctions and lower urinary tract symptoms due to benign prostatic hyperplasia. Eur Urol. 2011;60(4):809-825.

2. Gacci M, Corona G, Salvi M, et al. A systematic review and meta-analysis on the use of phosphodiesterase 5 inhibitors alone or in combination with $\alpha$-blockers for lower urinary tract symptoms due to benign prostatic hyperplasia. Eur Urol. 2012;61(5):994-1003.

3. Macfarlane GJ, Botto H, Sagnier PP, Teillac P, Richard F, Boyle P. The relationship between sexual life and urinary condition in the French community. J Clin Epidemiol. 1996;49(10):1171-1176.

4. Laumann EO, Paik A, Rosen RC. Sexual dysfunction in the United States: prevalence and predictors. JAMA. 1999;281(6):537-544.

5. Blanker MH, Bohnen AM, Groeneveld FP, et al. Correlates for erectile and ejaculatory dysfunction in older Dutch men: a community-based study. J Am Geriatr Soc. 2001;49(4):436-442.
6. Li MK, Garcia LA, Rosen R. Lower urinary tract symptoms and male sexual dysfunction in Asia: a survey of ageing men from five Asian countries. BJU Int. 2005;96(9):1339-1354.

7. Brookes ST, Link CL, Donovan JL, McKinlay JB. Relationship between lower urinary tract symptoms and erectile dysfunction: results from the Boston Area Community Health Survey. J Urol. 2008;179(1):250-255.

8. Sak SC, Hussain Z, Johnston C, Eardley I. What is the relationship between male sexual function and lower urinary tract symptoms (LUTS)? Eur Urol. 2004;46(4):482-487.

9. Rosen R, Altwein J, Boyle P, et al. Lower urinary tract symptoms and male sexual dysfunction: the multinational survey of the aging male (MSAM-7). Eur Urol. 2003;44(6):637-649.

10. Wein AJ, Coyne KS, Tubaro A, Sexton CC, Kopp ZS, Aiyer LP. The impact of lower urinary tract symptoms on male sexual health: EpiLUTS. BJU Int. 2009;103 Suppl 3:33-41.

11. Bouwman II, Van Der Heide WK, Van Der Meer K, Nijman R. Correlations between lower urinary tract symptoms, erectile dysfunction, and cardiovascular diseases: are there differences between male populations from primary healthcare and urology clinics? A review of the current knowledge. Eur J Gen Pract. 2009;15(3):128-135.

12. Andersson KE, de Groat WC, McVary KT, et al. Tadalafil for the treatment of lower urinary tract symptoms secondary to benign prostatic hyperplasia: pathophysiology and mechanism(s) of action. Neurourol Urodyn. 2011;30(3):292-301.

13. Filippi S, Morelli A, Sandner P, et al. Characterization and functional role of androgen-dependent PDE5 activity in the bladder. Endocrinology. 2007;148(3):1019-1029.

14. Fibbi B, Morelli A, Vignozzi L, et al. Characterization of phosphodiesterase type 5 expression and functional activity in the human male lower urinary tract. J Sex Med. 2010;7(1 Pt 1):59-69.

15. Morelli A, Filippi S, Comeglio P, et al. Acute vardenafil administration improves bladder oxygenation in spontaneously hypertensive rats. $J \mathrm{Sex}$ Med. 2010;7(1 Pt 1):107-120.

16. Morelli A, Sarchielli E, Comeglio P, et al. Phosphodiesterase type 5 expression in human and rat lower urinary tract tissues and the effect of tadalafil on prostate gland oxygenation in spontaneously hypertensive rats. $J$ Sex Med. 2011;8(10):2746-2760.

17. Vignozzi L, Filippi S, Morelli A, et al. Cavernous neurotomy in the rat is associated with the onset of an overt condition of hypogonadism. J Sex Med. 2009;6(5):1270-1283.

18. Angulo J, Cuevas P, Fernández A, et al. Tadalafil enhances the inhibitory effects of tamsulosin on neurogenic contractions of human prostate and bladder neck. J Sex Med. 2012;9(9):2293-2306.

19. Morelli A, Filippi S, Sandner P, et al. Vardenafil modulates bladder contractility through cGMP-mediated inhibition of RhoA/Rho kinase signaling pathway in spontaneously hypertensive rats. J Sex Med. 2009;6(6):1594-1608.

20. Gacci M, Del Popolo G, Macchiarella A, et al. Vardenafil improves urodynamic parameters in men with spinal cord injury: results from a single dose, pilot study. J Urol. 2007;178(5):2040-2043.

21. Gacci M, Ierardi A, Rose AD, et al. Vardenafil can improve continence recovery after bilateral nerve sparing prostatectomy: results of a randomized, double blind, placebo-controlled pilot study. J Sex Med. 2010;7(1 Pt 1):234-243.

22. Fibbi B, Penna G, Morelli A, Adorini L, Maggi M. Chronic inflammation in the pathogenesis of benign prostatic hyperplasia. Int $J$ Androl. 2010;33(3):475-488.

23. Gacci M, Vignozzi L, Sebastianelli A, et al. Metabolic syndrome and lower urinary tract symptoms: the role of inflammation. Prostate Cancer Prostatic Dis. Epub November 20, 2012.

24. Kramer G, Mitteregger D, Marberger M. Is benign prostatic hyperplasia (BPH) an immune inflammatory disease? Eur Urol. 2007;51(5): 1202-1216.

25. Filippi S, Vignozzi L, Morelli A, et al. Testosterone partially ameliorates metabolic profile and erectile responsiveness to PDE5 inhibitors in an animal model of male metabolic syndrome. J Sex Med. 2009;6(12): 3274-3288. 
26. Vignozzi L, Morelli A, Filippi S, et al. Farnesoid X receptor activation improves erectile function in animal models of metabolic syndrome and diabetes. $J$ Sex Med. 2011;8(1):57-77.

27. Vignozzi L, Morelli A, Sarchielli E, et al. Testosterone protects from metabolic syndrome-associated prostate inflammation: an experimental study in rabbit. J Endocrinol. 2012;212(1):71-84.

28. Morelli A, Comeglio P, Filippi S, et al. Mechanism of action of phosphodiesterase type 5 inhibition in metabolic syndrome-associated prostate alterations: an experimental study in the rabbit. Prostate. 2012;73(4): $428-441$.

29. Morelli A, Comeglio P, Filippi S, et al. Testosterone and farnesoid $\mathrm{X}$ receptor agonist INT-747 counteract high fat diet-induced bladder alterations in a rabbit model of metabolic syndrome. J Steroid Biochem Mol Biol. 2012;132(1-2):80-92.

30. Maneschi E, Morelli A, Filippi S, et al. Testosterone treatment improves metabolic syndrome-induced adipose tissue derangements. $J$ Endocrinol. 2012;215(3):347-362.

31. Zenzmaier C, Sampson N, Pernkopf D, Plas E, Untergasser G, Berger P. Attenuated proliferation and trans-differentiation of prostatic stromal cells indicate suitability of phosphodiesterase type 5 inhibitors for prevention and treatment of benign prostatic hyperplasia. Endocrinology. 2010;151(8):3975-3984.

32. Penna G, Mondaini N, Amuchastegui S, et al. Seminal plasma cytokines and chemokines in prostate inflammation: interleukin 8 as a predictive biomarker in chronic prostatitis/chronic pelvic pain syndrome and benign prostatic hyperplasia. Eur Urol. 2007;51(2):524-533.

33. McVary KT, Roehrborn CG, Avins AL, et al. Update on AUA guideline on the management of benign prostatic hyperplasia. $J$ Urol. 2011;185(5): 1793-1803.

34. Hutchison A, Farmer R, Verhamme K, Berges R, Navarrete RV. The efficacy of drugs for the treatment of LUTS/BPH, a study in 6 European countries. Eur Urol. 2007;51(1):207-215, discussion 215-216.

35. Debruyne F, Koch G, Boyle P, et al. Comparison of a phytotherapeutic agent (Permixon) with an alpha-blocker (Tamsulosin) in the treatment of benign prostatic hyperplasia: a 1-year randomized international study. Eur Urol. 2002;41(5):497-506.

36. Zlotta AR, Teillac P, Raynaud JP, Schulman CC. Evaluation of male sexual function in patients with Lower Urinary Tract Symptoms (LUTS) associated with Benign Prostatic Hyperplasia (BPH) treated with a phytotherapeutic agent (Permixon), Tamsulosin or Finasteride. Eur Urol. 2005;48(2):269-276.

37. Chapple CR, Montorsi F, Tammela TL, Wirth M, Koldewijn E, Fernández Fernández E; European Silodosin Study Group. Silodosin therapy for lower urinary tract symptoms in men with suspected benign prostatic hyperplasia: results of an international, randomized, doubleblind, placebo- and active-controlled clinical trial performed in Europe. Eur Urol. 2011;59(3):342-352.

38. Nickel JC, Fradet Y, Boake RC, et al. Efficacy and safety of finasteride therapy for benign prostatic hyperplasia: results of a 2-year randomized controlled trial (the PROSPECT study). PROscar Safety Plus Efficacy Canadian Two year Study. CMAJ. 1996;155(9):1251-1259.

39. Roehrborn CG, Siami P, Barkin J, et al; CombAT Study Group. The effects of combination therapy with dutasteride and tamsulosin on clinical outcomes in men with symptomatic benign prostatic hyperplasia: 4-year results from the CombAT study. Eur Urol. 2010;57(1): $123-131$.

40. McVary KT, Roehrborn CG, Avins AL, et al. Guideline on the management of benign prostatic hyperplasia (BPH). In: American Urological Association Guideline: Management of BPH. Baltimore, MD: American Urological Association Education and Research; 2003.

41. Bouza C, López T, Magro A, Navalpotro L, Amate JM. Systematic review and meta-analysis of Transurethral Needle Ablation in symptomatic Benign Prostatic Hyperplasia. BMC Urol. 2006;6:14.

42. Oelke M, Bachmann A, Descazeaud A, et al. EAU guidelines on the treatment and follow-up of non-neurogenic male lower urinary tract symptoms, including benign prostatic obstruction. Eur Urol. 2013; [Epub ahead of print. March 2013.].
43. Roehrborn CG. Standard surgical interventions: TURP/TUIP/OPSU. In: Kirby RS, McConnell JD, Fitzpatrick JM, Roehrborn CG, Boyle P, editors. Textbook of Benign Prostatic Hyperplasia. Oxford: ISIS Medical Media; 1996:341-378.

44. Briganti A, Naspro R, Gallina A, et al. Impact on sexual function of holmium laser enucleation versus transurethral resection of the prostate: results of a prospective, 2-center, randomized trial. J Urol. 2006;175(5): 1817-1821.

45. McVary KT, Roehrborn CG, Kaminetsky JC, et al. Tadalafil relieves lower urinary tract symptoms secondary to benign prostatic hyperplasia. J Urol. 2007;177(4):1401-1407.

46. Roehrborn CG, McVary KT, Elion-Mboussa A, Viktrup L. Tadalafil administered once daily for lower urinary tract symptoms secondary to benign prostatic hyperplasia: a dose finding study. $J$ Urol. 2008;180(4):1228-1234.

47. Bechara A, Romano S, Casabé A, et al. Comparative efficacy assessment of tamsulosin vs tamsulosin plus tadalafil in the treatment of LUTS/ BPH. Pilot study. $J$ Sex Med. 2008;5(9):2170-2178.

48. Liguori G, Trombetta C, De Giorgi G, et al. Efficacy and safety of combined oral therapy with tadalafil and alfuzosin: an integrated approach to the management of patients with lower urinary tract symptoms and erectile dysfunction. Preliminary report. J Sex Med. 2009;6(2): 544-552.

49. Porst H, McVary KT, Montorsi F, et al. Effects of once-daily tadalafil on erectile function in men with erectile dysfunction and signs and symptoms of benign prostatic hyperplasia. Eur Urol. 2009;56(4): $727-735$.

50. Roehrborn CG, Kaminetsky JC, Auerbach SM, Montelongo RM, Elion-Mboussa A, Viktrup L. Changes in peak urinary flow and voiding efficiency in men with signs and symptoms of benign prostatic hyperplasia during once daily tadalafil treatment. BJU Int. 2010;105(4): 502-507.

51. Dmochowski R, Roehrborn C, Klise S, Xu L, Kaminetsky J, Kraus S. Urodynamic effects of once daily tadalafil in men with lower urinary tract symptoms secondary to clinical benign prostatic hyperplasia: a randomized, placebo controlled 12-week clinical trial. J Urol. 2010;183(3):1092-1097.

52. Porst H, Kim ED, Casabé AR, et al; LVHJ study team. Efficacy and safety of tadalafil once daily in the treatment of men with lower urinary tract symptoms suggestive of benign prostatic hyperplasia: results of an international randomized, double-blind, placebo-controlled trial. Eur Urol. 2011;60(5):1105-1113.

53. Donatucci CF, Brock GB, Goldfischer ER, et al. Tadalafil administered once daily for lower urinary tract symptoms secondary to benign prostatic hyperplasia: a 1-year, open-label extension study. BJU Int. 2011;107(7):1110-1116.

54. Oelke M, Giuliano F, Mirone V, Xu L, Cox D, Viktrup L. Monotherapy with tadalafil or tamsulosin similarly improved lower urinary tract symptoms suggestive of benign prostatic hyperplasia in an international, randomised, parallel, placebo-controlled clinical trial. Eur Urol. 2012;61(5):917-925.

55. Regadas RP, Reges R, Cerqueira JB, et al. Urodynamic effects of the combination of tamsulosin and daily tadalafil in men with lower urinary tract symptoms secondary to benign prostatic hyperplasia: a randomized, placebo-controlled clinical trial. Int Urol Nephrol. 2012;45(1): 39-43.

56. Yokoyama O, Yoshida M, Kim SC, et al. Tadalafil once daily for lower urinary tract symptoms suggestive of benign prostatic hyperplasia: a randomized placebo- and tamsulosin-controlled 12-week study in Asian men. Int J Urol. Epub 2012 September 7, 2012.

57. Liu L, Zheng S, Han P, Wei Q. Phosphodiesterase-5 inhibitors for lower urinary tract symptoms secondary to benign prostatic hyperplasia: a systematic review and meta-analysis. Urology. 2011;77(1):123-129.

58. Martínez-Salamanca JI, Carballido J, Eardley I, et al. Phosphodiesterase type 5 inhibitors in the management of non-neurogenic male lower urinary tract symptoms: critical analysis of current evidence. Eur Urol. 2011;60(3):527-535. 
Research and Reports in Urology

\section{Publish your work in this journal}

Research and Reports in Urology is an international, peer-reviewed, open access journal publishing original research, reports, editorials, reviews and commentaries on all aspects of adult and pediatric urology in the clinic and laboratory including the following topics: Pathology, pathophysiology of urological disease; Investigation and treatment of

urological disease; Pharmacology of drugs used for the treatment of urological disease. The manuscript management system is completely online and includes a very quick and fair peer-review system, which is all easy to use. Visit http://www.dovepress.com/testimonials.php to read real quotes from published authors.

Submit your manuscript here: http://www.dovepress.com/research-and-reports-in-urology-journal 